\title{
On the Construction of Energy-Efficient Broadcast and Multicast Trees in Wireless Networks
}

\author{
Jeffrey E. Wieselthier \\ Information Technology Division \\ Naval Research Laboratory \\ Washington, DC 20375 \\ wieselthier@itd.nrl.navy.mil
}

\author{
Gam D. Nguyen \\ Information Technology Division \\ Naval Research Laboratory \\ Washington, DC 20375 \\ nguyen@itd.nrl.navy.mil
}

\author{
Anthony Ephremides \\ Electrical Engineering Dept. and \\ Institute of Systems Research \\ University of Maryland \\ College Park, MD 20742 \\ tony@eng.umd.edu
}

\begin{abstract}
The wireless networking environment presents formidable challenges to the study of broadcasting and multicasting problems. After addressing the characteristics of wireless networks that distinguish them from wired networks, we introduce and evaluate algorithms for tree construction in infrastructureless, all-wireless applications. The performance metric used to evaluate broadcast and multicast trees is energyefficiency. We develop the Broadcast Incremental Power Algorithm, and adapt it to multicast operation as well. This algorithm exploits the broadcast nature of the wireless communication environment, and addresses the need for energy-efficient operation. We demonstrate that our algorithm provides better performance than algorithms that have been developed for the link-based, wired environment.
\end{abstract}

\section{INTRODUCTION}

In this paper, we study the problems of broadcasting and multicasting in all-wireless networks. Most previous research and development work on multicasting has centered on tethered, point-to-point (typically high speed) networks. By contrast, we address infrastructureless (peer-to-peer) applications, and we incorporate the broadcast properties of wireless communication media into our algorithms and performance measures. Among the most crucial issues related to mobile wireless applications is that of operation in limited-energy environments. Our focus is on the development of algorithms for the formation of energyefficient trees for broadcast and multicast communication.

Our approach to energy-efficient communication departs from the traditional layered structure in that we jointly address the issues of transmitted power levels (and hence network connectivity, a Physical layer function) and multicast tree formation (a routing function, associated with the Network layer). We argue that such joint decisions on connectivity and routing can result in significant improvement in energy efficiency, as compared to a rigid layered structure that makes these decisions independently. ${ }^{1}$ Moreover, our approach is based on the "node-based" nature of wireless communications. By contrast, previously developed models for multicasting have been based on "linkbased" models, which, although appropriate for wired applications, do not reflect the properties of the all-wireless network environment.

Our focus is on source-initiated broadcasting (one-to-all) and multicasting (one-to-many) of "session" (or connectionoriented) traffic. In either case, our objective is to form a

\footnotetext{
This work was supported by the Office of Naval Research.

${ }^{1}$ Here we consider only the energy used for transmission, neglecting for the present the energy associated with reception and signal processing; the joint study of all forms of energy expenditure and the associated trade-offs are not considered here.
}

minimum-energy tree, rooted at the source, that reaches all of the desired destinations. We demonstrate the fundamental differences between wired and wireless networks, and introduce a new node-based broadcast algorithm that takes into account wireless network properties. We show how this algorithm can also serve as the basis for a multicasting algorithm, and we demonstrate its superior performance, as compared to schemes that are based on more-conventional, link-based approaches.

A crucial issue in wireless networks is the trade-off between the "reach" of wireless transmission (namely the simultaneous reception by many nodes of a transmitted message) and the resulting interference by that transmission. We assume that the power level of a transmission can be chosen within a given range of values. Therefore, there is a trade-off between reaching more nodes in a single hop by using higher power (but at a higher interference cost) versus reaching fewer nodes in that single hop by using lower power (but at a lower interference cost). Another crucial issue is that of energy consumption, because of the nonlinear attenuation properties of radio signals.

Few studies have addressed multicasting specifically for wireless networks. For example, the problem of multicast scheduling in cellular mobile networks was studied in [1], and a forwarding multicast protocol for noncellular networks was studied in [2]. Although [3] addressed the multicasting problem with a goal toward reaching efficient and nearminimum-cost algorithms for wireless networks, their approach was link-based, and hence does not take into consideration the node-based nature of wireless communications. Most other multicasting studies have been limited to the case of stationary networks that are not wireless (e.g., [4], [5], [6]).

To assess the complex trade- offs one at a time, we assume in this paper that there is no mobility. Nevertheless, the impact of mobility can be incorporated into our models because transmitter power can be adjusted to accommodate the new locations of the nodes, as necessary. In other words, the capability to adjust transmission power provides considerable "elasticity" to the topological connectivity, and hence may reduce the need for hand-offs and tracking. We also assume the availability of a large number of bandwidth resources (i.e., unlimited number of frequencies or time slots or orthogonal CDMA codes), so that contention for the channel is not an issue. Moreover, we assume that sufficient transceiver resources are available at each of the nodes in the network; thus, calls are never blocked because of the unavailability of either a transmitter or receiver. Under these assumptions, we can focus on the determination of minimumcost (in our case, minimum-energy) broadcast and multicast trees. The problem we address involves the designation of which nodes are to transmit, and the power levels at which they are to do so. Once broadcasting and multicasting 
models are developed for node-based models, future studies can address the impact of mobility and limited resources (both bandwidth and equipment), as well as protocol issues associated with determining connectivity and reserving resources.

\section{ARCHITECTURAL ISSUES IN ALL-WIRELESS NETWORKS}

The all-wireless networks studied here are quite different from the cellular systems and wireless LANs that have been developed in the commercial domain. Cellular systems have fixed base stations, which communicate among themselves using dedicated non-wireless lines; thus, the only multicast problems that are new in those systems involve tracking the mobile users. Otherwise, wireless communication is limited to that between mobile users and base stations. In fullyconnected wireless LANs, since there is single-hop connectivity among all the nodes, the multicasting problem is trivial. However, in ad hoc wireless networks it is possible to establish a link between any pair of nodes, provided that each has a transceiver available for this purpose and that the signal-to-noise ratio at the receiving node is sufficiently high. Thus, unlike the case of wired networks, the set of network links and their capacities are not determined a priori, but depend on factors such as distance between nodes, transmitted power, error-control schemes, other-user interference, and background noise. Thus, even when the physical locations of the nodes are fixed, many of the factors that affect network topology (and hence network control schemes) are (at least partially) influenced by the actions of the network nodes. Furthermore, in ad hoc networks no distinction can be made between uplink and downlink traffic, thus greatly complicating the interference environment. Therefore, the wireless networking environment poses many new challenges not encountered in non-wireless or cellular networks, even when mobility is not addressed.

In this paper, we focus on wireless networks in which the node locations are fixed, and the channel conditions unchanging. The wireless channel is distinguished by its broadcast nature; when omnidirectional antennas are used, every transmission by a node can be received by all nodes that lie within its communication range. Consequently, if the multicast group membership includes multiple nodes in the immediate communication vicinity of the transmitting node, a single transmission suffices for reaching all these receivers.

In addition to interference, another undesirable impact of the use of high transmitter power is that it results in increased energy usage. Since the propagation loss varies nonlinearly with distance (at somewhere between the second and fourth power), in unicast applications it is best (from the perspective of transmission energy consumption) to transmit at the lowest possible power level, even though doing so requires multiple hops to reach the destination. However, in multicast applications it is not prudent to draw such conclusions a priori because the use of higher power may permit simultaneous connectivity to a sufficiently large number of nodes, so that the total energy required to reach all members of the multicast group may be actually reduced. Furthermore, even for unicast applications, the use of lower power (and, hence, multiple hops) necessitates the complex coordination of more signals and therefore may actually result in higher total energy expenditure.

Thus, the choice of transmitted power levels depends ultimately on complex trade-offs between energy limitations and the demands of protocol operation. In view of the complex interdependencies among many aspects of network design (e.g., transmitted power levels, signal processing considerations, spectral efficiency, mobility effects, etc.), the traditional layered architectures proposed for protocol design may not provide satisfactory performance. Therefore, it may be beneficial to design protocols that span several of the traditional layers to address appropriately the unique characteristics of the all-wireless environment [7], [8]. Our studies do, in fact, support this conjecture.

\section{WiRELESS COMMUNICATIONS MODEL}

We consider source-initiated, circuit-switched, multicast sessions. The network consists of $N$ nodes, which are randomly distributed over a specified region. Each node has several transceivers, and can thus support several multicast sessions simultaneously. Any node is permitted to initiate multicast sessions. Multicast requests and session durations are generated randomly at the network nodes. Each multicast group consists of the source node plus at least one destination node. Additional nodes may be needed as relays either to provide connectivity to all members of the multicast group or to reduce overall energy consumption or both. The set of nodes that support a multicast session (the source node, all destination nodes, and all relay nodes) is referred to as a multicast tree.

The connectivity of the network depends on the transmission power. We assume that each node can choose its power level, not to exceed some maximum value $p_{\max }$. The nodes in any particular multicast tree do not necessarily have to use the same power levels; moreover, a node may use different power levels for the various multicast trees in which it participates.

We assume that the received signal power varies as $r^{-\alpha}$, where $r$ is the range and $\alpha$ is a parameter that typically takes on a value between 2 and 4, depending on the characteristics of the communication medium. Based on this model the transmitted power required to support a link between two nodes separated by range $r$ is proportional to $r^{\alpha}$. Without loss of generality, we set the normalizing constant equal to 1 , resulting in:

$$
\begin{aligned}
p_{i j} & =\text { power needed for link between Node } i \text { and Node } j \\
& =r^{\alpha},
\end{aligned}
$$

where $r$ is the distance between Node $i$ and Node $j$. If the maximum permitted transmitter power $p_{\max }$ is sufficiently large, the nodes will be able to transmit at sufficiently high power so that the network is fully connected. ${ }^{2}$

We assume the use of omnidirectional antennas; thus all nodes within communication range of a transmitting node can receive its transmission. It is important to note how the broadcast property of wireless communication can be exploited in multicast applications. Consider the example shown in Fig. 1, in which a subset of the multicast tree involves Node $i$, which is transmitting to its neighbors, Node $j$ and Node $k$. The power required to reach Node $j$ is $P_{i j}$ and the power required to reach Node $k$ is $P_{i k}$. A single transmission at power $P_{i,(j, k)}=\max \left\{P_{i j}, P_{i k}\right\}$ is sufficient to reach both Node $j$ and Node $k$, based on our assumption of

\footnotetext{
${ }^{2}$ We assume that the communication medium is uniform, i.e., $\alpha$ is constant throughout the region of interest, there are no obstacles (such as buildings or mountains), and that the region is totally flat (hence no line-of-sight limitations resulting from the earth's curvature).
} 
omnidirectional antennas. The ability to exploit this property of wireless communication, which we refer to as the "wireless multicast advantage," makes multicasting an excellent setting in which to study the potential benefits of energy-efficient protocols.

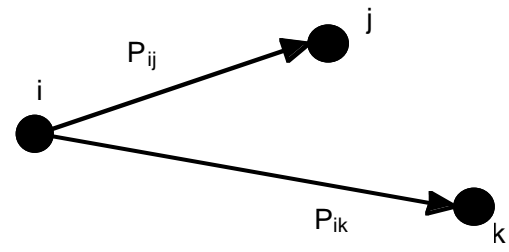

Fig. 1 - The "wireless multicast advantage:" $P_{i, j, k)}=\max \left\{P_{i j}, P_{i k}\right\}$.

As a result of the wireless multicast advantage, the correct view of the omnidirectional wireless communication medium is as a node-based environment that is characterized by the following properties:

- A node's transmission is capable of reaching another node if the latter is within communication range, which in turn means that the received signal-to-interferenceplus-noise ratio exceeds a given threshold and that the receiving nodes have allocated (scheduled) receiver resources for this purpose.

- The total power required to reach a set of other nodes is simply the maximum required to reach any of them individually.

By contrast, in wired models, as long as there is a wire or cable link connecting two nodes, the reception is ensured over that link, and the cost of Node $i$ 's transmission to Node $j$ and Node $k$ would be the sum of the costs to the individual nodes. ${ }^{3}$ Thus, wired networks can be viewed correctly as link-based.

\section{MINIMUM-ENERGY BROADCAST TREES}

We first address the problem of constructing the minimum-energy, source-based broadcast tree for each newly arriving broadcast session request. Doing so involves the choice of transmitter-power levels and relay nodes. As noted earlier, we address only the transmission energy. Thus, the total energy of the broadcast tree is simply the sum of the energy expended at each of the transmitting nodes in the tree; leaf nodes (which do not transmit) do not contribute to this quantity. Since we are considering session traffic, all transmitting nodes transmit for the entire duration of each session. Therefore, the total transmission energy is proportional to the total power needed to maintain the tree. Hence, we evaluate performance in terms of the total power required to maintain the tree.

We assume throughout this paper that ample bandwidth is available, and that each node has a sufficient number of transceivers to accommodate all service requests. An insufficient quantity of either of these resources can result in

\footnotetext{
${ }^{3}$ In wired networks, energy is not a concern; the cost of a link would typically be related to bandwidth and congestion (and hence delay) considerations. The case of wireless applications with highly directive antennas is similar to the case of wired networks in the sense that multiple beams may be needed to reach multiple destinations; thus the total cost of a node's transmissions to its neighbors would be equal to the sum of the cost of the individual beams needed to reach each individual destination.
}

the construction of trees that do not reach all destinations, use more than the minimum energy (because only suboptimal trees can be constructed), or both.

We start with a simple example with two destinations, and extended our approach to larger examples by means of a recursive technique. Our examples in this section are based on the broadcasting problem, in which all nodes in the network (other than, of course, the source) are destinations. In Section VI we return to the problem of multicasting in which only a subset of the network nodes must be reached, while non-destination nodes may be used as relays. Including such nodes may result in reduced overall power consumption, or perhaps in providing a connected network where one was not achievable without the use of such relays.

We emphasize a crucial difference between wired and wireless networks. In wired networks, the broadcasting problem can be formulated as the well-known minimum-cost spanning tree (MST) problem. This formulation is based on the existence of a cost associated with each link in the network; the total cost of the broadcast tree is the sum of the link costs. The situation in wireless networks is different, however, because of the "wireless multicast advantage" property, discussed in Section III, which permits all nodes within communication range to receive a transmission without additional expenditure of transmitter power. Therefore, the standard MST problem, which reflects the link-based nature of wired networks, does not capture the node-based nature of wireless networks. We do not know of any scalable solutions to the node-based version of this problem. ${ }^{4}$ In this paper we introduce a heuristic that takes into account the wireless multicast advantage in the formation of low-energy broadcast trees. We use such lowenergy broadcast trees (including versions based on both link-based and node-based versions) as the basis for some of our heuristics for the construction of suboptimal multicast trees in wireless networks.

\section{A. Minimum-Energy Broadcasting: 2 Destinations}

We consider a source node $\mathrm{S}$ (located at the origin) and two destination nodes $\mathrm{D}_{1}$ (located on the $x$-axis, without loss of generality) and $\mathrm{D}_{2}$, as shown in Fig. 2. The coordinates of $\mathrm{D}_{1}$ and $\mathrm{D}_{2}$ determine the angle $\theta$. The distance between $\mathrm{S}$ and $\mathrm{D}_{1}$ is $r_{1}$, the distance between $\mathrm{S}$ and $\mathrm{D}_{2}$ is $r_{2}$, and the distance between $\mathrm{D}_{1}$ and $\mathrm{D}_{2}$ is $r_{12}$. We assume, without loss of generality, that $r_{2}>r_{1}$ and define:

$P_{\mathrm{S} 1}=r_{1}^{\alpha}=$ power needed for link between $\mathrm{S}$ and $\mathrm{D}_{1}$

$P_{\mathrm{S} 2}=r_{2}^{\alpha}=$ power needed for link between $\mathrm{S}$ and $\mathrm{D}_{2}$

$P_{12}=r_{12}^{\alpha}=$ power needed for link between $\mathrm{D}_{1}$ and $\mathrm{D}_{2}$ In this simple example, there are two alternative strategies:

a) $S$ transmits using $P_{\mathrm{S} 2}$ : both $\mathrm{D}_{1}$ and $\mathrm{D}_{2}$ are reached.

b) $S$ transmits using $P_{\mathrm{S} 1}$ : only $\mathrm{D}_{1}$ is reached.

$\mathrm{D}_{1}$ then transmits to $\mathrm{D}_{2}$ with power $P_{12}$, resulting in a total power of $P_{\mathrm{S} 1}+P_{12}$.

4 It was recently shown that the related (although somewhat different) problem of finding a minimal-size multipoint relay set in wireless networks is NP-complete [10]. 


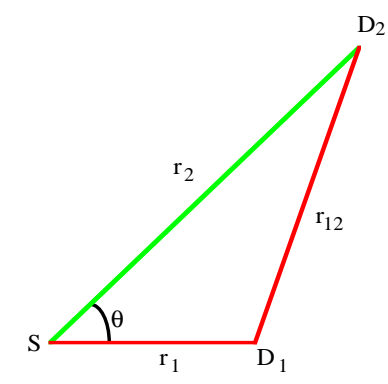

Fig. 2 - Broadcasting to two destinations.

We would like to choose the alternative that results in the smaller value of total power consumption. For the case of propagation that follows a $1 / r^{2}$ law, it is very simple to derive the following result from simple geometrical considerations:

- Use strategy (a) if $r_{1}>r_{2} \cos \theta$,

- Use strategy (b) otherwise.

For the general case of propagation behaving as $1 / r^{\alpha}$, algebraic manipulation results in the following:

- Use strategy (a) if

$$
x^{\alpha}-1<\left(1+x^{2}-2 x \cos \theta\right)^{\frac{\alpha}{2}},
$$

where $x=r_{2} / r_{1}$;

- Use strategy (b) otherwise.

This result is shown graphically in Fig. 3. For example, in the region above the curve (for each particular value of $\alpha$ ) it is best to use strategy (a). It is of special interest to note that for $\alpha \geq 3$ (which is characteristic of many realistic environments) the boundary separating these regions is quite steep; therefore a simple heuristic that uses strategy (a) whenever $\theta \geq 90^{\circ}$ and strategy (b) otherwise should be expected to provide nearly optimal performance. Thus, the incentive to use the shortest available links increases as $\alpha$ increases.

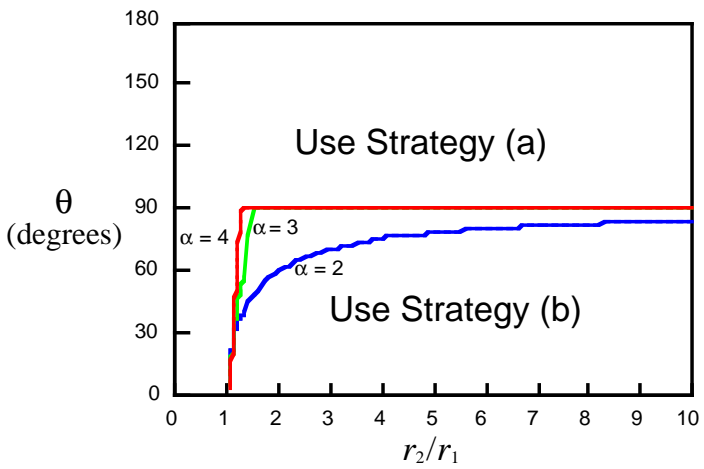

Fig. 3 - Minimum-energy broadcasting strategies to two destinations $\left(r_{2} \geq r_{1}\right)$; each curve represents the strategy for a particular value of $\alpha$.

\section{B. A Recursive Formulation}

The minimum-energy broadcasting problem becomes more interesting as the number of destinations increases. It is difficult to make generalizations about the desirability of using the shortest available links because the use of a higher power transmission can often result in the ability to reach several nodes with a single transmission, thereby resulting in lower overall power in the complete tree.
A brute-force approach for the determination of minimum-energy trees is an exhaustive search over all possible trees. However, the number of alternative strategies increases rapidly as the number of destinations increases, making such an approach impractical, except for small networks. Nevertheless, the effects of complexity can be mitigated somewhat by means of the recursive formulation described in [11]. We denote the distance between the Source and Destination $D_{n}$ to be $r_{n}$. Without loss of generality, we assume that $r_{1}<r_{2}<\ldots<r_{N}$.

For example, let us consider the case of three destinations. The source has three alternatives, namely to transmit with enough power to reach only $D_{1}$, both $D_{1}$ and $D_{2}$, or all three destinations. If the source reaches only $\mathrm{D}_{1}$, it effectively delegates to $D_{1}$ the responsibility of relaying to $D_{2}$ and $D_{3}$. One can thus remap the origin to the location of $\mathrm{D}_{1}$, and use the already obtained solution to the problem of broadcasting to two destinations in the evaluation of strategies for the three-destination example. If the Source transmits with enough power to reach both $D_{1}$ and $D_{2}$, then one of these nodes must relay to $\mathrm{D}_{3}$. An exhaustive search procedure would evaluate the total power under all of these alternatives, and pick the one that results in the minimum total power.

In general, the solution for $N_{D}$ destinations can be expressed in terms of the solutions for various subsets of the solutions for a smaller number of destinations. Unfortunately, the complexity of this formulation is high, making it impractical except for small networks. One way to roughly estimate complexity is to evaluate the number of times that the solution for the two-destination problem is called during the course of the algorithm. For the case of four destinations, it is called three times, which is certainly easy to handle. However, the number of calls to this subproblem increases rapidly as $N_{D}$ increases; e.g., for $N_{D}=$ 10 , more than 51,000 calls are needed, and for $N_{D}=13$ more than 14 million calls are needed. Nevertheless, this approach may serve as the basis for a suboptimal heuristic that provides less than an exhaustive search of all possible trees.

\section{THE BroAdCAST InCREMENTAL POWER AlgorithM}

Our objective is the determination of the minimum-power tree, rooted at the source node, that reaches all of the other nodes in the network. For wireless networks, this is a difficult problem for which no scalable solutions appear to be available at this time. Therefore, it is necessary to develop heuristics. The total power associated with the tree is simply the sum of the powers at all transmitting nodes. Clearly, this is a node-based (rather than link-based) metric because it enables us to exploit the wireless multicast advantage. Nevertheless, some of the algorithms we have studied are based on the minimization of link-based costs (as a heuristic for the minimization of the true, node-based cost).

In this section, we introduce and describe the Broadcast Incremental Power (BIP) Algorithm, which is the major contribution of this paper. We also discuss two additional approaches, which are based on conventional networking techniques. Unlike the other two approaches, BIP exploits the wireless multicast advantage in the construction of the broadcast tree. 
In all of the algorithms studied here, each transmission by a node is characterized by its transmitter power level, as well as a designation of which (possibly several) of the nodes receiving this transmission are to forward it toward which of the ultimate destination nodes. In small examples, we are able to compare our results to those obtained by an exhaustive search algorithm that is based on the recursive procedure discussed in [10].

\section{A. Description of BIP}

We describe the basic operation of BIP here by presenting a simple example of tree construction. The objective is to construct a minimum-cost (in our case, minimum-power) tree, rooted at the Source.

Step 1: Fig. 4(a) shows a ten-node network, in which Node 10 is the Source. A propagation constant of $\alpha=2$ is assumed. Initially, the tree consists of only the Source. We begin by determining the node that the Source can reach with minimum expenditure of power, i.e., the Source's nearest neighbor, which is Node 9 . This node is added to the tree. Thus, at this point, two nodes are included in the tree, namely Node 10 and Node 9 (Fig. 4(a)). The notation $10 \rightarrow 9$ means that the addition to the tree at this step is the transmission from Node 10 to Node 9.

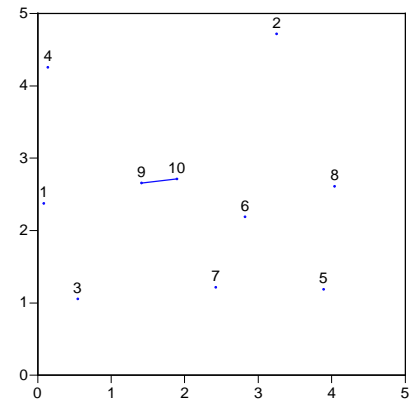

(a) Step 1: $10 \rightarrow 9$

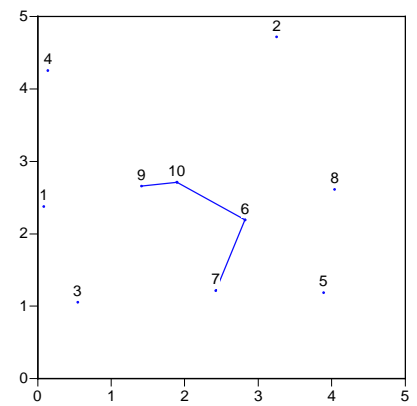

(c) Step 3:6 $\rightarrow 7$

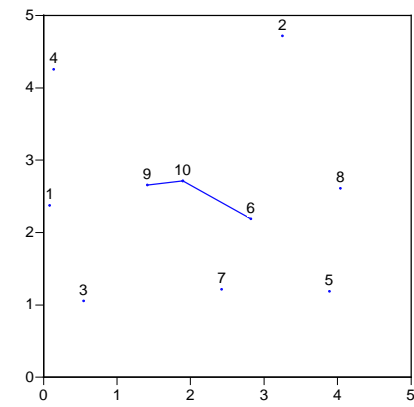

(b) Step 2: $10 \rightarrow 6$

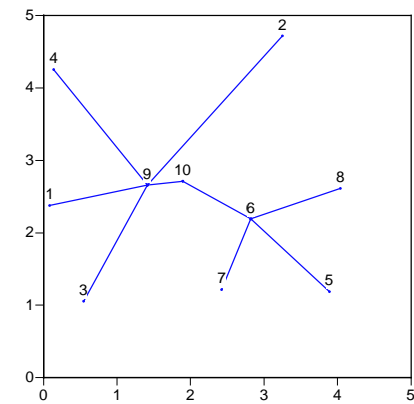

(d) final tree $(P=10.90)$

Fig. 4 - Example of tree construction using BIP (first three steps and final tree); $\alpha=2$.

Step 2: We then determine which "new" node can be added to the tree at minimum additional cost. There are two alternatives. Either Node 10 can increase its power to reach a second node, or Node 9 can transmit to its nearest neighbor that is not already in the tree. In this example, Node 10 increases its power level to reach Node 6 (Fig. 4(b)). Note that the cost associated with the addition of Node 6 to the tree is the incremental cost associated with increasing Node 10's power from a level sufficient to reach Node 9 to a level sufficient to reach Node 6 . The cost of a transmission between Node 10 and Node 9 is $r_{10.9}^{\alpha}$, and the cost of a transmission between Node 10 and Node 6 is $r_{10,6}^{\alpha}$. The incremental cost associated with adding Node 6 to the preliminary tree consisting of Node 9 and Node 10 is $r_{10,9}^{\alpha}-$ $r_{10,6}^{\alpha}$. We are able to exploit the wireless multicast advantage because both Node 6 and Node 9 can be reached when Node 10 transmits with sufficient power to reach Node 6 .

Step 3: There are now three nodes in the tree, namely Node 6, Node 9, and Node 10. For each of these nodes, we determine the incremental cost to reach a new node. (Since Node 6 and Node 9 were not previously transmitting, their respective incremental costs will equal their full transmission powers if they are chosen to transmit; since Node 10 was already transmitting, its incremental cost is only the required increase in its transmission power). The node that can be added with minimum incremental cost (which turns out to be Node 7) is added to the tree (Fig. 4(c)).

Continue: This procedure is continued until all nodes are included in the tree, as shown in Fig. 4(d). The order in which the nodes were added in steps 4 through 9 is: $6 \rightarrow 8$, $6 \rightarrow 5,9 \rightarrow 1,9 \rightarrow 3,9 \rightarrow 4,9 \rightarrow 2$.

Evaluation of Transmitted Power: The total power required to maintain this tree is the sum of the transmitted powers at each of the transmitting nodes. In this example, Nodes 10, 6, and 9 transmit, while the other nodes, which are leaf nodes, do not. The overall transmitter power is therefore

$$
\begin{aligned}
P= & \max \left(r_{10,6}^{\alpha}, r_{10,9}^{\alpha}\right)+\max \left(r_{6,5}^{\alpha}, r_{6,7}^{\alpha}, r_{6,8}^{\alpha}\right) \\
& +\max \left(r_{9,1}^{\alpha}, r_{9,2}^{\alpha}, r_{9,3}^{\alpha}, r_{9,4}^{\alpha}\right) \\
= & r_{10,6}^{\alpha}+r_{6,5}^{\alpha}+r_{9,2}^{\alpha}
\end{aligned}
$$

For the present case of $\alpha=2$, we have $P=10.90$.

BIP is similar in principle to Prim's algorithm for the formation of MSTs, in the sense that new nodes are added to the tree one at a time (on a minimum-cost basis) until all nodes are included in the tree. In fact, the implementation of this algorithm is based on the standard Prim algorithm, with one fundamental difference. Whereas the inputs to Prim's algorithm are the link costs $P_{i j}$ (which remain unchanged throughout the execution of the algorithm), BIP must dynamically update the costs at each step (i.e., whenever a new node is added to the tree) to reflect the fact that the cost of adding new nodes to a transmitting node's list of neighbors is the incremental cost. Consider an example in which Node $i$ is already in the tree (it may be either a transmitting node or a leaf node), and Node $j$ is not yet in the tree. For all such Nodes $i$ (i.e., all nodes already in the tree), and Nodes $j$ (i.e., nodes not yet in the tree), the following is evaluated:

$$
P_{i j}^{\prime}=P_{i j}-P(i) \text {, }
$$

where $P_{i j}$ is the link-based cost of a transmission between Node $i$ and Node $j$ (i.e., it is $r_{i j}^{\alpha}$ ), and $P(i)$ is the power level at which Node $i$ is already transmitting (prior to the addition of Node $j$; if Node $i$ is currently a leaf node, $P(i)=0$ ). The quantity $P_{i j}^{\prime}$ represents the incremental cost associated with adding Node $j$ to the set of nodes to which Node $i$ already transmits. The pair $\{i, j\}$ that results in the minimum value of $P_{i j}^{\prime}$ is selected, i.e., Node $i$ transmits at a power level sufficient to reach Node $j$. Thus, one new node is added to the tree at every step of the algorithm. 
Unlike Prim's algorithm, which guarantees the formation of minimum-cost spanning trees for link-based costs (as in wired networks), BIP does not necessarily provide minimumcost trees for wireless networks. However, neither do any other scalable algorithms that we are aware of. The performance results of Section 7 demonstrate nonetheless that this algorithm does, in fact, provide satisfactory performance.

\section{B. Broadcast Algorithms based on Link-Based Techniques}

Two of the algorithms we have studied are based on wellknown techniques, namely the use of shortest unicast paths and the use of spanning trees, both of which use link-based costs.

\section{1) Broadcast Least-Unicast-cost (BLU) Algorithm}

A straightforward (but far from optimal) approach is the use of broadcast trees that consist of the superposition of the best unicast paths to each individual destination (see e.g., [11]). It is assumed that an underlying unicast algorithm (such as the Bellman-Ford or Dijkstra algorithm) provides "minimum-distance" paths from the source node to every other node. Since BLU is based on the use of a scalable unicast algorithm, it also is scalable.

Also note that, although algorithms based on minimumdistance paths are normally used for packet-switched applications, we are using this approach here for sessionoriented traffic, since a cost (involving power and possibly congestion) can be defined for each link in the network. By contrast, in circuit-switched wired applications it is difficult to define a link cost because energy is not of concern and because delay is not an appropriate metric (as it would be in packet-switched applications) since resources are reserved in circuit-switched applications. Instead, blocking probability is the only overall objective, and there is no known way of mapping that objective to individual link metrics. Summarizing the above, we have:

BLU: A minimum-cost path from the source node to every other node is established. The broadcast tree consists of the superposition of these unicast paths.

Fig. 5(a) shows the multicast tree produced by BLU for the same example shown in Fig. 4. The power required to maintain this tree is $P=12.17$. Note that whereas both Node 9 and Node 10 transmit with relatively high power under BLU, only Node 9 transmits with relatively high power under BIP. Consequently, the overall power consumption under BIP is somewhat lower for this example. Thus, the failure of BLU to exploit the wireless multicast advantage results in higher overall power expenditure.

\section{2) Broadcast Link-based MST (BLiMST) Algorithm}

This algorithm is based on the use of the standard MST formulation (as in wired networks) in which a link cost is associated with each pair of nodes (i.e., the power to sustain the link). Thus, the "wireless multicast advantage" is ignored in the construction of the MST. Since the MST problem is of polynomial complexity, it is scalable. Once the MST is constructed in this manner, the evaluation of its cost (i.e., the total power needed to sustain the broadcast tree) does take into consideration the wireless multicast advantage. Summarizing the above, we have:
BLiMST: A minimum-cost (minimum-power) spanning tree is formed using standard (link-based) MST techniques.

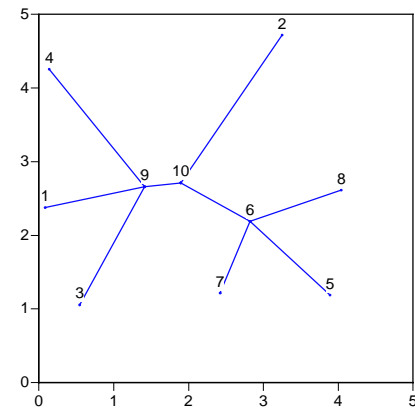

(a) $\operatorname{BLU}(P=12.17)$

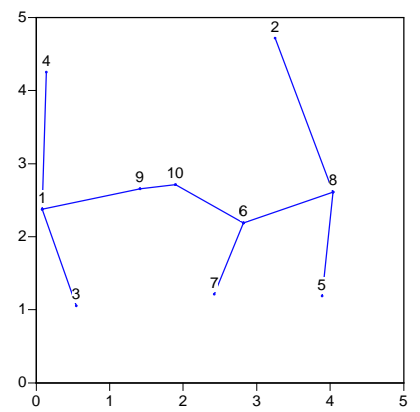

(b) BLiMST $(P=13.22)$
Fig. 5 - Trees produced by link-based algorithms $(\alpha=2)$.

Fig. 5(b) shows the multicast tree produced by BLiMST for the same example shown in Fig. 4. The power required to maintain this tree is $P=13.22$. Similarly to the case of BLU, the failure of BLiMST to exploit the wireless multicast advantage results in higher overall power expenditure.

\section{Complexity Considerations}

The complexity of BLU, when implemented by means of the Dijkstra algorithm, is $O\left(N^{2}\right)$, where $N$ is the number of nodes in the network [12] (p. 111).

The complexity of BLiMST, when implemented by means of Prim's algorithm, is $O\left(N^{3}\right)$ when a straightforward implementation is used [12] (p. 524). However, a moresophisticated implementation using a Fibonacci heap yields complexity $O(M+N \log N)=O\left(N^{2}\right)$, where $M=N(N-1) / 2$ is the number of links (in a fully connected network).

Since BIP is based on Prim's algorithm, it also has complexity $O\left(N^{3}\right)$. Because of the need to update the costs $P_{i j}^{\prime}$ at each step of the algorithm, it is not yet clear whether the Fibonacci heap technique is applicable here.

\section{The Sweep: Removing Unnecessary Transmissions}

The performance of the algorithms presented here can be improved by eliminating unnecessary transmissions by means of what we call the "sweep" operation. Before describing the procedure, we show two examples. Fig. 6(a) shows the tree that results from applying the sweep operation to the tree produced by BIP (Fig. 4(d)). In this example, Node 9 and Node 6 serve as relays. Nodes 1, 2, 3, 4, 5, 7, and 8 are leaf nodes; thus they do not transmit, and hence do not contribute to the power consumption. It is easy to see (by simple geometry, since we assume the use of omnidirectional antennas) that Node 9's transmitted power is sufficient to reach Node 6; thus Node 10 can reduce its power so that it reaches only Node 9. By doing so, the overall power consumption is reduced from $P=10.90$ to $P=10.00$.

Fig. 6(b) shows a sweep example based on BLU. Again, Node 9 and Node 6 serve as relays and Nodes 1, 2, 3, 4, 5, 7, and 8 are leaf nodes. The total transmitter power required to maintain this tree is $P=12.17$. It is easy to see that the transmission by Node 10 (which is sufficient to reach Node 2) can also reach Nodes 1, 3, and 4 without further expenditure of power. Therefore, the transmission by Node 9 is unnecessary. The resulting power consumption is reduced from $P=12.17$ to $P=8.00$. 
The sweep procedure is summarized as follows. We examine the nodes in ascending ID order, i.e., from 1 through $N .^{5}$ Leaf nodes are ignored because they do not transmit. The non-leaf node with the lowest ID is Node 6, whose downstream neighbors are Nodes 5, 7, and 8; thus Node 6 is the first candidate for restructuring. Since the transmission by Node 6 does not reach any neighbors of Nodes 5, 7, and 8, no changes are made here. The situation at Node 9 is similar. Finally, we reach Node 10 . As noted above, Node 10 reaches all of Node 9's downstream neighbors; therefore, the transmission by Node 9 can be eliminated. ${ }^{6}$

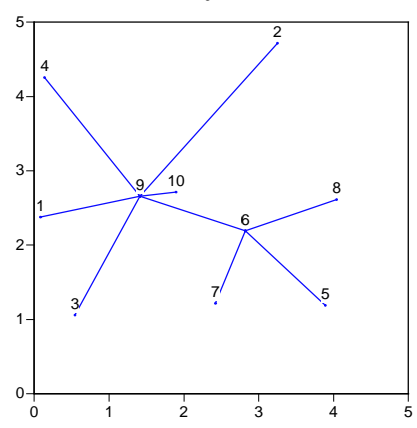

(a) $\operatorname{BIP}(P=10.00)$

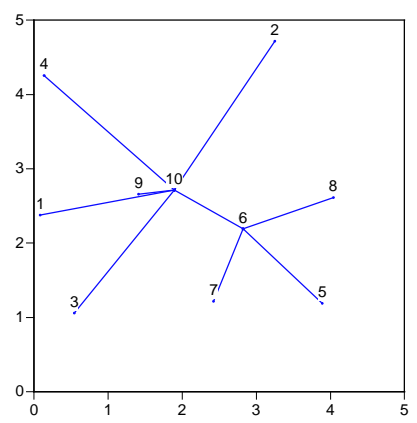

(b) BLU $(P=8.00)$
Fig. 6 - Post-sweep trees produced by BIP and BLU for the network of Fig. $4(\alpha=2)$.

We have applied the same sweep procedure to all of our algorithms. Typically, a single application of the sweep operation provides significant improvement; small further improvement can often be obtained by repeating the sweep once more, but little improvement has been found by additional applications of this procedure. In this particular example, BLU provides a lower power post-sweep tree than BIP; however, in most cases (see Section 7) the tree produced by BIP has lower power, both before and after the sweep. BLiMST provides a tree with $P=13.22$ before the sweep, with no improvement obtained by sweeping in this particular network.

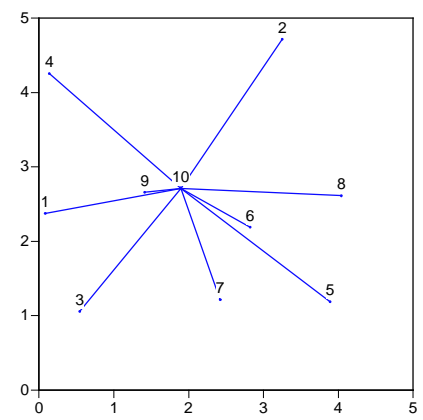

Fig. 7 - Optimal multicast tree for our example network for $\alpha=2(P=6.30)$

\section{E. The Optimal Tree}

The optimal (lowest power) tree, obtained by exhaustive search, is a hub network (Fig. 7), in which the Source (Node

\footnotetext{
5 Alternative schemes for ordering the sequence in which the nodes are examined are currently under investigation, including schemes that start from the Source and progress outwardly along the tree.

${ }^{6}$ In some cases, the sweep operation discovers opportunities to reduce transmitted power (although without eliminating a transmission entirely), namely when the responsibility of relaying to a particular node can be transferred to another node.
}

10) transmits with sufficient power to reach all of the other nodes; the resulting tree power is $P=6.30$. In most of our examples for which the true optimal solution is available (i.e., ten-node networks), however, the performance provided by the three algorithms we have studied is much closer to that of the optimal solution than in this particular example.

\section{AlgORITHMS FOR MUlTiCASTING}

It is well known that the determination of a minimum-cost multicast tree in wired networks is a difficult problem, which can be modeled as the NP-complete Steiner tree problem. This problem appears to be at least as hard in wireless networks as it is in wired networks. As we noted earlier, we know of no scalable algorithms for the minimum-energy broadcast problem. Thus, heuristics are definitely needed.

In this section we discuss three of the multicasting algorithms we have studied. They are direct analogs of the broadcasting algorithms discussed in Section 5, and "Multicast" replaces "Broadcast" in their names.

\section{Multicast Incremental Power (MIP) Algorithm}

A broadcast tree is formed using BIP. To obtain the multicast tree, the broadcast tree is pruned by eliminating all transmissions that are not needed to reach the members of the multicast group. More specifically, nodes with no downstream destinations will not transmit, and some nodes will be able to reduce their transmitted power (i.e., if their more-distant downstream neighbors have been pruned from the tree).

\section{Multicast Least-Unicast-cost (MLU) Algorithm}

This is identical to BLU, except that unicast paths are established only to the desired destinations rather than to all network nodes. The multicast tree consists of the superposition of the appropriate unicast paths.

\section{Multicast Link-based MST (MLiMST) Algorithm}

A broadcast tree is formed using BLiMST. As with MIP, to obtain the multicast tree, the broadcast tree is pruned by eliminating all transmissions that are not needed to reach the members of the given multicast group.

\section{PERFORMANCE RESUlts}

We have evaluated the performance of the three algorithms for many network examples. Networks with a specified number of nodes (typically 10 or 100) are randomly generated within a square region (e.g., $5 \times 5$, as in Fig. 4), i.e., the location of each node is randomly generated. One of the nodes is randomly chosen to be the Source. Multicast groups of a specified size are chosen randomly from the overall set of nodes. Since broadcasting is simply a special case of multicasting in which all of the nodes are included in the multicast group, we discuss our results for broadcasting and multicasting together and refer solely to the multicasting versions of the algorithms. No restrictions are placed on the maximum transmitter power (i.e., $p_{\max }=\infty$ ). The transmitter power actually used $\left(r^{\alpha}\right)$ depends on the distance $(r)$ to the farthest neighbor to which a node is transmitting. We have considered propagation loss exponents of $\alpha=2$ and $\alpha=4$. In all cases, (i.e., for a specified network size, multicast group size, and tree algorithm), our results are based on the performance of 100 randomly generated networks. 
Our performance metric is the total power of the multicast (or broadcast) tree. To facilitate the comparison of our algorithms over a wide range of network examples, we use the notion of the normalized power for each network example. For each individual network example, say network $m$, we specify the locations of the nodes, the Source, and the destinations, and we compute the power associated with the multicast tree generated by each of the algorithms. To determine a benchmark for each network instance, we define

$$
\begin{aligned}
Q_{i}(m)= & \text { total power of multicast tree for } \\
& \text { network } m, \text { generated by algorithm } i,
\end{aligned}
$$

and

$$
Q_{\text {best }}(m)=\min \left\{Q_{i}(m), i \in I\right\},
$$

where $I$ is the set of algorithms. Thus, $Q_{\text {best }}(m)$ is the power of the lowest-power tree among the set of algorithms (for the particular network instance $m$ ). For our larger (100-node network) examples, the set $I$ consists of Algorithms 1,2 , and 3. For our small (ten-node network) examples, we also have results for a fourth algorithm, namely the recursive exhaustive search algorithm [10], which provides the optimal solution; thus $Q_{\text {best }}(m)$ is the true value of the power of the optimal tree in such cases. We then define the normalized power associated with algorithm $i$ to be

$$
Q_{i}^{\prime}(m)=\frac{Q_{i}(m)}{Q_{\text {best }}(m)} .
$$

This metric provides a measure of how close each algorithm comes to providing the lowest-power tree. In all cases, we have used the performance values obtained after a single application of the Sweep Algorithm, discussed in Section 5.

Note: Consider a $5 \times 5$ square unit region in which nodes are randomly distributed (such as Fig. 4). It is important to note that the units of distance used in this study are arbitrary. For example, the doubling of all distances in the $5 \times 5$ region while the nodes maintain their relative positions (resulting in nodes being distributed in a $10 \times 10$ square unit region), results in link power levels that increase by a factor of $2^{\alpha}$. Since the resulting trees produced by the algorithms studied here remain the same under any scaling of the distance, the overall tree power levels also increase by the same factor of $2^{\alpha}$. Therefore, any ratio comparisons of the tree powers produced by the algorithms are independent of the distance scaling factor. In other words, the normalized power (5) does not depend on the size of the region, even though the absolute power does.

Table 1 summarizes performance for the three algorithms for networks with ten nodes, various multicast-group sizes, and for a propagation constant value of $\alpha=2$. Each entry represents the results for 100 randomly generated networks. The entries in the table are mean and variance, respectively, of the normalized tree power $Q_{i}^{\prime}(m)$. The multicast-group size includes the source node in addition to the destination nodes. For the case of a single destination (group size $=2$ ), MLU provides not only the best average performance, but also the best performance for every network instance (it provides the same solution as the exhaustive search). This is certainly not surprising because this is simply the unicast routing problem, for which the Dijkstra algorithm provides the optimal solution. For a group size of 5, MLU still provides the best performance in terms of mean tree power; MIP provides a slightly higher mean value, but a smaller variance. For the case of broadcasting (group size $=10$ ),
MIP provides the best performance, both in terms of mean and variance. In fact, MIP performs better than MLiMST for all group sizes.

TABLE 1 - Mean and Variance of Normalized Tree Power For 100 NETWORK INSTANCES: 10-NODE NETWORKS, $\alpha=2$.

\begin{tabular}{|l|c|c|c|}
\hline $\begin{array}{c}\text { Group } \\
\text { size }\end{array}$ & MLU & MLiMST & MIP \\
\hline 2 & $1.0000 ; 0.0000$ & $1.1256 ; 0.1244$ & $1.0583 ; 0.0201$ \\
\hline 5 & $1.1040 ; 0.0245$ & $1.1852 ; 0.0415$ & $1.1055 ; 0.0193$ \\
\hline 10 & $1.1388 ; 0.0270$ & $1.1987 ; 0.0471$ & $1.1049 ; 0.0145$ \\
\hline
\end{tabular}

Table 2 provides similar results for $\alpha=4$. MIP again provides improved performance, as compared to the other two algorithms, for group sizes of 5 and greater.

TABLE 2 - Mean and Variance of Normalized Tree Power for 100 NeTWORK InSTANCES: 10-NODE NETWORKS, $\alpha=4$.

\begin{tabular}{|l|c|c|c|}
\hline $\begin{array}{c}\text { Group } \\
\text { size }\end{array}$ & MLU & MLiMST & MIP \\
\hline 2 & $1.0000 ; 0.0000$ & $1.0381 ; 0.0315$ & $1.0285 ; 0.0209$ \\
\hline 5 & $1.0401 ; 0.0120$ & $1.0430 ; 0.0124$ & $1.0283 ; 0.0091$ \\
\hline 10 & $1.0473 ; 0.0098$ & $1.0474 ; 0.0128$ & $1.0229 ; 0.0050$ \\
\hline
\end{tabular}

Performance results for 100-node networks are shown in Tables 3 and 4 . As noted above, the normalization is taken with respect to the best of the three algorithms under study because the true optimal values are not available. As in the case of the smaller networks, MLU performs better than the other two algorithms when multicast groups are small. This is not surprising, since this algorithm provides a minimumcost path to each individual destination. By contrast, the other two algorithms are based on the formation of a minimum-cost tree that reaches all nodes (with subsequent pruning to eliminate paths to nodes that are not in the multicast group), and therefore may provide long, circuitous paths to some of the destinations. For $\alpha=2$, MIP provides the best performance for group sizes of 25 or greater; for $\alpha=$ 4 , MIP is best for group sizes of 10 or more. MIP again provides better performance than MLiMST in all cases, as it did in the ten-node network examples.

TABle 3 - Mean and Variance of Normalized Tree Power for 100 NETWORK INSTANCES: 100-NODE NETWORKS, $\alpha=2$

\begin{tabular}{|l|l|l|c|}
\hline $\begin{array}{c}\text { Group } \\
\text { size }\end{array}$ & \multicolumn{1}{|c|}{ MLU } & \multicolumn{1}{c|}{ MLiMST } & MIP \\
\hline 5 & $1.0410 ; 0.0072$ & $1.2711 ; 0.0757$ & $1.1892 ; 0.0440$ \\
\hline 10 & $1.0680 ; 0.0088$ & $1.1496 ; 0.0153$ & $1.0729 ; 0.0161$ \\
\hline 25 & $1.1060 ; 0.0097$ & $1.0831 ; 0.0057$ & $1.0135 ; 0.0010$ \\
\hline 50 & $1.1495 ; 0.0103$ & $1.0801 ; 0.0020$ & $1.0025 ; 0.0001$ \\
\hline 75 & $1.1502 ; 0.0047$ & $1.0683 ; 0.0019$ & $1.0009 ; 0.0000$ \\
\hline 100 & $1.1456 ; 0.0044$ & $1.0676 ; 0.0019$ & $1.0010 ; 0.0000$ \\
\hline
\end{tabular}

Our performance results indicate that our proposed algorithm, MIP, provides better performance than MLiMST over the complete range of network examples that we have studied, based on the criteria of mean tree power as well as variance. We attribute this improved performance to the fact that MIP exploits the node-based wireless multicast advantage property, whereas MLiMST ignores this property as it forms trees on the basis of link-based costs. Both MIP 
and MLiMST provide better performance than MLU when the size of the multicast groups is moderate to large. When multicast groups are large, the structure obtained by first establishing a broadcast tree is highly beneficial. However, when multicast groups are small, many energy-inefficient paths are established; such behavior would be expected even for truly optimal broadcast trees, and is a consequence of the suboptimal nature of the pruning operation. Our observations apply also for $\alpha=4$. As $\alpha$ increases, the penalty for using longer links increases; thus trees may consist of a larger number of shorter links, but this fact does not change the relative behavior of the algorithms.

TABLE 4 - Mean and Variance of Normalized Tree Power for 100 NETWORK INSTANCES: 100-NODE NETWORKS, $\alpha=4$.

\begin{tabular}{|l|c|c|c|}
\hline $\begin{array}{c}\text { Group } \\
\text { size }\end{array}$ & \multicolumn{1}{|c|}{ MLU } & \multicolumn{1}{c|}{ MLiMST } & MIP \\
\hline 5 & $1.0623 ; 0.0178$ & $1.1211 ; 0.0309$ & $1.1058 ; 0.0223$ \\
\hline 10 & $1.1123 ; 0.0246$ & $1.0570 ; 0.0064$ & $1.0409 ; 0.0051$ \\
\hline 25 & $1.1243 ; 0.0154$ & $1.0359 ; 0.0027$ & $1.0116 ; 0.0006$ \\
\hline 50 & $1.1531 ; 0.0128$ & $1.0253 ; 0.0006$ & $1.0030 ; 0.0001$ \\
\hline 75 & $1.1673 ; 0.0153$ & $1.0198 ; 0.0005$ & $1.0027 ; 0.0001$ \\
\hline 100 & $1.1615 ; 0.0105$ & $1.0232 ; 0.0006$ & $1.0016 ; 0.0000$ \\
\hline
\end{tabular}

Figures $8-11$ illustrate graphically the relative performance of the algorithms we have studied. The horizontal axis represents the network ID ( $m$ ranges between 1 and 100), and the vertical axis is the normalized tree power $Q_{i}^{\prime}(m)$. These results, which correspond to a subset of those presented in Table 3, permit us to evaluate the relative performance of the algorithms for a set of 100 network instances. The dotted curves represents the case of MLU; it often provides the best performance for small multicast groups, but this advantage decreases and eventually disappears as the size of the multicast group increases. Its performance is usually the worst of the three algorithms for large multicast group sizes, although there is one case in which it provides the best performance for the broadcast case.

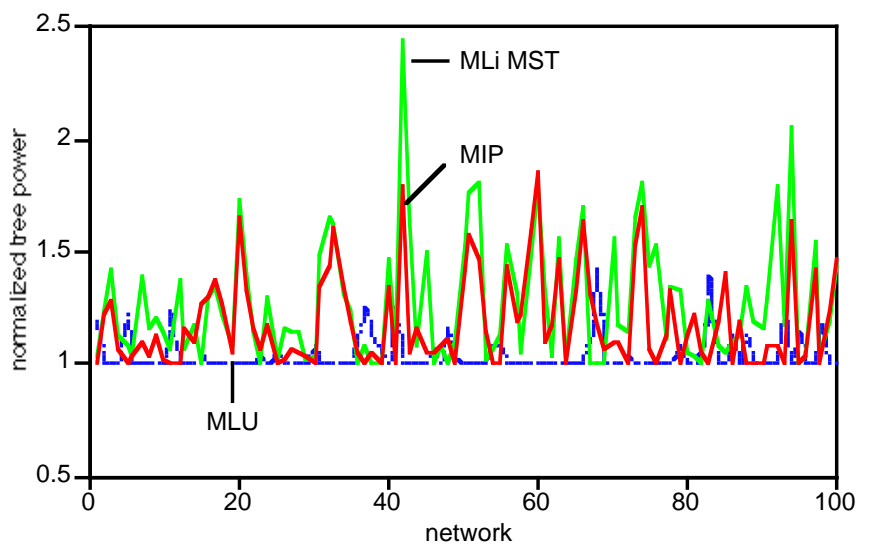

Fig. 8 - Normalized tree power for 100 network instances; 100 -node networks, multicast group size $=5, \alpha=2$.

The lighter solid curve represents MLiMST, and the darker solid curve represents MIP. For most network instances, MIP performs better than MLiMST, with the advantage increasing substantially as the size of the multicast groups increases. For large multicast group sizes, MIP provides the best performance in almost all network instances.

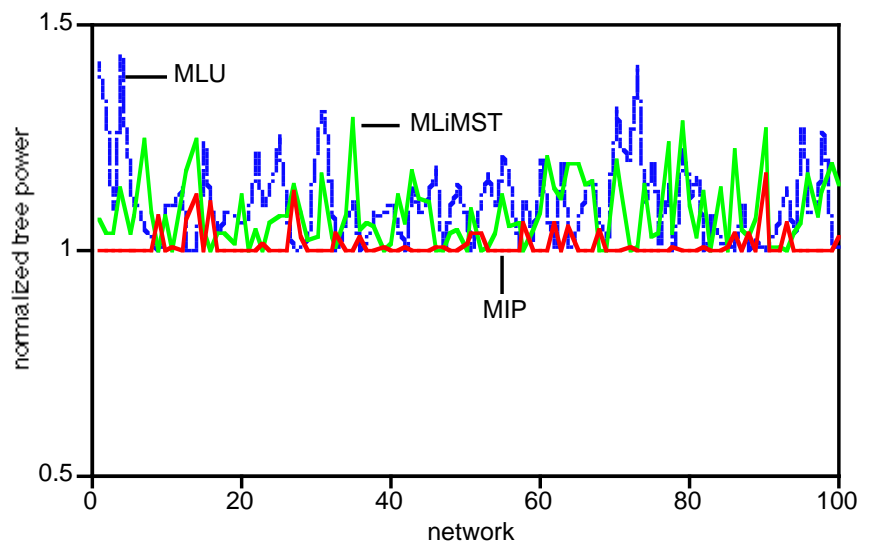

Fig. 9 - Normalized tree power for 100 network instances; 100 -node networks, multicast group size $=25, \alpha=2$.

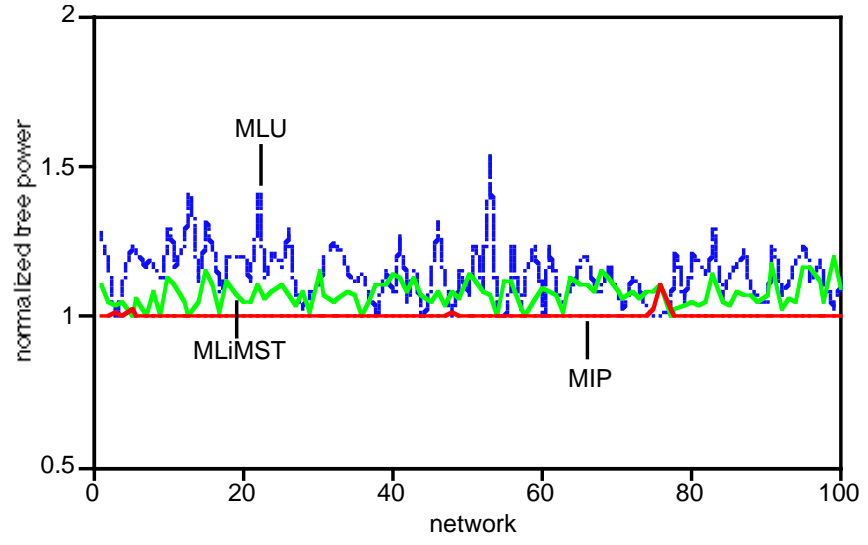

Fig. $10-$ Normalized tree power for 100 network instances; 100 -node networks, multicast group size $=50, \alpha=2$.

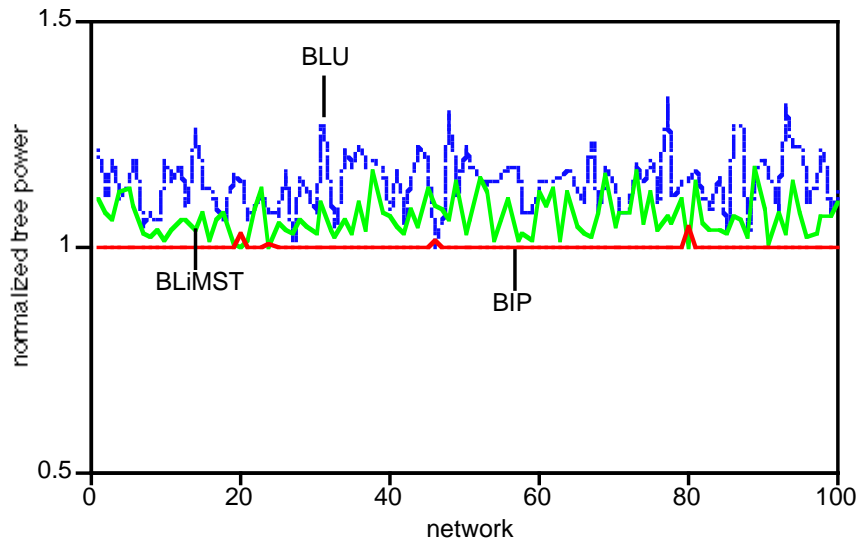

Fig. 11 - Normalized tree power for 100 network instances; 100 -node networks, broadcast case (i.e., group size $=100$ ), $\alpha=2$.

Thus far, we have discussed only the normalized values of tree power. This metric is useful for the comparison of the relative performance of our algorithms. Additional insight into the properties of the algorithms can be obtained by looking at the actual values of tree power. Table 5 shows the mean value of the tree power for the same set of network instances summarized in Table 3 for a $5 \times 5$ region. As noted 
earlier, the actual communication ranges (rather than their relative values) are relevant to the evaluation of absolute values of power, but not to the normalized values. Also shown, in parentheses below the mean value, are the minimum and maximum values of tree power observed over the set of 100 network instances for each of the three algorithms. Table 6 shows the corresponding results for the examples of Table 4 . The network that results in the minimum (or maximum) value of tree power is typically different for the three algorithms.

TABLE 5 - Mean Tree Power (And Minimum and Maximum Values) FOR 100 NETWORK INSTANCES:

100-Node Networks, $5 \times 5$ Region, $\alpha=2$ (See Table 3 ).

\begin{tabular}{|c|c|c|c|}
\hline $\begin{array}{c}\text { Group } \\
\text { size }\end{array}$ & MLU & MLiMST & MIP \\
\hline 5 & $\begin{array}{l}3.983 \\
(1.716, \quad 8.017)\end{array}$ & $\begin{array}{ll}4.818 & \\
(1.775, & 7.735)\end{array}$ & $\begin{array}{l}4.511 \\
(1.737, \quad 7.618)\end{array}$ \\
\hline 25 & $\begin{array}{l}8.819 \\
(6.157,11.014) \\
\end{array}$ & \begin{tabular}{|ll}
8.631 & \\
$(6.437$, & $10.626)$ \\
\end{tabular} & $\begin{array}{l}8.096 \\
(6.162,9.868) \\
\end{array}$ \\
\hline 50 & $\begin{array}{l}10.769 \\
(8.360, \quad 13.683)\end{array}$ & $\begin{array}{ll}10.135 & \\
(7.696, & 12.603)\end{array}$ & $\begin{array}{l}9.415 \\
(7.118,11.792)\end{array}$ \\
\hline 100 & $\begin{array}{l}12.348 \\
(10.149,14.926)\end{array}$ & $\begin{array}{ll}11.514 & \\
(9.762, & 13.323)\end{array}$ & $\begin{array}{l}10.802 \\
(9.004,12.592)\end{array}$ \\
\hline
\end{tabular}

TABLE 6 - Mean Tree Power (And Minimum and Maximum Values) FOR 100 NETWORK INSTANCES:

100-Node Networks, $5 \times 5$ Region, $\alpha=4$ (See Table 4 ).

\begin{tabular}{|c|c|c|c|}
\hline $\begin{array}{l}\text { Group } \\
\text { size }\end{array}$ & MLU & MLiMST & MIP \\
\hline 5 & $\begin{array}{l}1.129 \\
(0.269, \quad 2.398)\end{array}$ & $\begin{array}{ll}1.180 \\
(0.337, & 2.389)\end{array}$ & $\begin{array}{ll}1.164 \\
(0.337, \quad 2.361)\end{array}$ \\
\hline 25 & $\begin{array}{l}2.552 \\
(1.534,5.611)\end{array}$ & $\begin{array}{ll}2.352 \\
(1.410, & 4.473)\end{array}$ & $\begin{array}{l}2.300 \\
(1.393,4.472)\end{array}$ \\
\hline 50 & $\begin{array}{l}3.137 \\
(1.975,5.214)\end{array}$ & $\begin{array}{ll}2.797 \\
(1.709, & 4.584)\end{array}$ & $\begin{array}{ll}2.738 \\
(1.645, & 4.461)\end{array}$ \\
\hline 100 & $\begin{array}{l}3.792 \\
(2.530,9.436)\end{array}$ & $\begin{array}{ll}3.351 \\
(2.239, & 9.373)\end{array}$ & $\begin{array}{ll}3.282 & \\
(2.188, & 9.325)\end{array}$ \\
\hline
\end{tabular}

The mean tree power increases as the multicast group size increases. This is certainly expected, since a greater number of destinations must be reached. The ratio of maximum to minimum values decreases as the group size increases. This is also expected, because when the group size is small, the minimum power tree depends not only on the random locations of the complete set of 100 nodes, but also on which nodes are included in the group.

The values of tree power depend on the units of distance. Recall that the transmitted power is defined to be $r^{\alpha}$, where we have arbitrarily defined the units of $r$ such that all nodes are randomly located with a $5 \times 5$ square region; scaling $r$ to a different set of units would result in considerably different values. In our examples, the values of tree power are lower for $\alpha=4$ than for $\alpha=2$. This is a consequence of the fact that most of the communication ranges are less than 1 (recall that the 100 nodes are randomly located in a $5 \times 5$ region). Also, there is a significantly greater variation in the ratio of maximum to minimum values for $\alpha=4$. This results from the greater cost of longer links for higher values of $\alpha$.

\section{CONCLUSIONS}

In this paper, we have identified some of the fundamental issues associated with energy-efficient broadcasting and multicasting in infrastructureless wireless networks, and we have presented preliminary algorithms for the solution of this problem. Our studies show that improved performance can be obtained when exploiting the properties of the wireless medium; i.e., networking schemes should reflect the nodebased nature of wireless communications, rather than simply adapt link-based schemes originally developed for wired networks. In particular, the Broadcast Incremental Power (BIP) Algorithm, which exploits the wireless multicast advantage, provides better performance than the other algorithms we have studied over a wide range of network examples.

Furthermore, the fact that improved performance can be obtained by jointly considering physical layer issues and network layer issues suggests that novel approaches to wireless networking, which incorporate the vertical integration of protocol layer functions, may provide advantages over traditional network architectures. A major challenge, and a topic of continued research, is the development of distributed algorithms that provide the benefits that have been demonstrated in this paper. Furthermore, it is important to study the impact of limited bandwidth and transceiver resources, as well as to develop mechanisms to cope with node mobility.

\section{REFERENCES}

[1] M. Nagy and S. Singh, "Multicast scheduling algorithms in mobile networks," Cluster Computing, 1, pp. 177-185, December 1998.

[2] C.-C. Chiang, M. Gerla, and L. Zhang, "Forwarding group multicast protocol (FGMP) for multihop, mobile wireless networks," Cluster Computing, 1, pp. 187-196, December 1998.

[3] K. Makki, N. Pissinou, and O. Frieder, "Efficient solutions to multicast routing in communication networks," Mobile Networks and Applications (MONET), 1, pp. 221-232, 1996.

[4] C. Diot, W. Dabbous, and J. Crowcroft, "Multipoint communication: a survey of protocols, functions, and mechanisms," IEEE Journal on Selected Areas in Communications, 15, pp. 277-290, April 1997.

[5] M. H. Ammar, G. C. Polyzos, and S. K. Tripathi, "Special issue on network support for multipoint communication," IEEE Journal on Selected Areas in Communications, 15, April 1997.

[6] L. Zhang, S. Deering, D. Estrin, S. Shenker, and D. Zappala, "RSVP: a new resource reservation protocol," IEEE Network Magazine, pp. 8-18, September 1993.

[7] NSF, "Research priorities in wireless and mobile communications and networking, report of a workshop," March 1997.

[8] C. D. Rais and S. K. Tripathi, "Studying vertical dependence to improve NFS performance in wireless networks," Cluster Computing, 1, pp. 225-235, December 1998.

[9] L. Viennot, "Complexity results on election of multipoint relays in wireless networks," Report RR-3584, INRIA, December 1998.

[10] J. E. Wieselthier, G. D. Nguyen, and A. Ephremides, "Algorithms for energy-efficient multicasting in static ad hoc wireless networks," Mobile Networks \& Applications (MONET), in press.

[11] D. Bertsekas and R. Gallager, Data Networks, Englewood Cliffs: Prentice Hall, 1992.

[12] R. K. Ahuja, T. L. Magnanti, and J. B. Orlin, Network Flows, Englewood Cliffs: Prentice Hall, 1993. 